\title{
The Effects of Task Types on Listening Test Performance:
}

\section{A Retrospective Study}

\author{
Yo In'nami \\ Doctoral Course, University of Tsukuba
}

\begin{abstract}
1 Introduction
The increasing interest in outcome-based approaches to assessment in language testing (e.g., Brindley, 1998; McKay, 2000) has heightened the need for more research on fair testing by which more valid inferences can be drawn. Among many variables related to test performance, of particular interest are the effects of task types (Chapelle, 1998; Kunnan, 2000). Research in this area is divided into two approaches, quantitative (i.e., focusing on products, or test scores) and qualitative (i.e., focusing on test-taking processes). First, quantitative studies on the comparability of task types (Berne, 1992; In'nami, 2001, 2004; Shohamy, 1984; Wolf, 1991) have shown that multiple choice tasks are easier than open ended tasks, multiple choice tasks are easier than summary cloze tasks, and the difficulty of open ended and summary cloze tasks is statistically not different. Quantitative approaches also compare the trait or construct equivalence of multiple choice and open ended tasks, and find out that when the two task types are constructed using the same stem, correlations between them approach unity (Rodriguez, 2003). Second, qualitative studies (Alderson, 1990; Buck, 1991; Ross, 1997; Storey, 1997; Wu, 1998; Yamashita, 2003) have overall shown that different test-takers engage in different test-taking processes and thus the same item may measure different ability depending on test-takers. Both quantitative and qualitative results suggest that task types influence students' test performance.

Despite determined efforts to investigate how task types affect test performance, past studies are limited in two ways. First, most studies (Berne, 1992; In'nami, 2001, 2004; Kobayashi, 1995; Rodriguez, 2003; Shohamy, 1984; Wolf, 1991) take only quantitative approaches to this issue. Since quantitative studies do not usually examine how task types function to produce effects on test performance, qualitative studies are also necessary. Although a few studies use qualitative methods (i.e., verbal protocol analysis), they focus on independent task types (open ended tasks, Alderson, 1990, Buck, 1991; matching tasks, Ross, 1997; cloze tasks, Storey, 1997; multiple choice tasks, Wu, 1998; gap-filling tasks, Yamashita, 2003) and do not compare multiple task types directly. This makes it difficult to investigate the effects of task types on test performance, because each study is separately conducted under different conditions (e.g., text contents, text organization, item types) and thus variables other than task types are not systematically controlled.
\end{abstract}


Second, although Riley and Lee (1996) compare recall and summary tasks, idea units (Carrell, 1985; Kroll, 1977) are used as text comprehension units. Idea units are widely used mostly in L2 studies, but there seems to be little evidence that idea units are the processing units of text comprehension.

The purpose of the present research is to examine qualitatively the effects of three task types (i.e., multiple choice, open ended, and summary cloze tasks) on listening test performance, taking the two limitations of the previous studies into account. The present study specifically addresses how task types affect the cognitive processes of taking listening tests. The current study surmounts the first limitation of the past studies by testing the three tasks qualitatively and concurrently in one study, whereas it overcomes the second limitation by conducting propositional analysis (e.g., Kintsch, 1998) on retrospective verbal protocol data. Three research questions examined are (a) whether test scores are different across the three task types, (b) whether the number of propositions used that are related to the task types in answering test items is different across the three task types, and (c) whether the number of propositions used that are related to the listening text in answering test items is different across the three task types. Although the current study focuses on the latter two research questions, the research question 1 is added for comparison with the findings of previous quantitative studies. Test-taking processes in the current study refer to processes test-takers use in pre- and while-listening, and answering questions.

\section{Method}

\subsection{Participants}

A total of 45 Japanese EFL (English as a Foreign Language) students participated in the study, the majority of them being 18-20 years of age and in the first, second, or third years of their university courses. Most of them had previously had six years of English language learning at secondary schools in Japan.

\subsection{Materials}

The Listening Performance Test was developed to measure the participants' listening performance in three types of tasks. Listening performance is based on the ability to "process extended samples of realistic spoken language, automatically and in real time," and "understand the linguistic information that is unequivocally included in the text" (Buck, 2001, p. 114).

One section of past examinations papers of the Key English Test (KET; University of Cambridge Local Examinations Syndicate [UCLES], 2001b, 2001c) was adapted for the present research. This section bonsisted of one text and five items, and was checked from validity aspects (Messick, 1996) using validation methods (Chapelle, 1999), and was found to offer acceptable validity of inferences (see In'nami, 2004). The test consisted of a one-and-half minute recorded dialogue. 
To ensure that the responses required for each item in one task corresponded as closely as possible to an item in each of the other tasks, a multiple choice task was selected first because it was considered to be more difficult to construct than open ended and summary cloze formats. Since UCLES follows strict guidelines to achieve a high standard of item production (UCLES, 2001a, p. 3), it may be possible to say that the multiple choice task was adequately constructed and appropriate for the current research purpose. Then, the multiple choice task was rewritten into open ended and summary cloze formats. For the open ended version, options in the multiple choice version were deleted, and some items were rephrased into an interrogative form.

For the summary cloze version, the following three steps were followed. First, the author and a graduate student majoring in language testing summarized the listening text independently, ensuring that the summaries contained the information required to answer the multiple choice and the open ended items. Disagreement was solved through discussion. Second, several gaps were inserted in these summaries by parallelizing the item contents with those in the other two task types. Third, the summary cloze task was checked by two native speakers of English for its appropriateness as the summary of listening text and for linguistic expressions in English.

It should be noted that the summaries were written in English (the participants' L2) for the following two reasons. First, if they were presented in the participants' L1 (Japanese) the participants would have been more likely to obtain information on the content by reading them instead of by listening to the aural texts. Second, it was necessary to be consistent with previous studies using the summary cloze format (Berne, 1992; Wolf, 1991). It is also worth adding that the summaries were designed to be as short but as natural as possible. This was because excessively long summaries would measure reading rather than listening performance, but at the same time excessively short summaries might make the summary cloze format too similar to the open ended format and thus its uniqueness would be lost.

After the three versions of the tasks were prepared, they were inspected by five non-native speakers of English for task equivalency, and the tasks were further revised. See Appendices for the Listening Performance Test.

\subsection{Procedures}

\subsubsection{Research Design}

The current study used a balanced, between-subject design in which equal numbers of the participants were randomly assigned to only one condition of the three task types ( $n=15$ for each condition). Results of a one-way analysis of variance (ANOVA) showed that the three groups were not statistically different in terms of their person ability estimates as measured by the listening proficiency test originally developed and validated in In'nami (2004), $F(2,42)=1.10, p=.34$. This test consisted of 38 items selected from the three levels (KET, Preliminary English Test, and First 
Certificate in English) of the past papers of the UCLES Main Suite. Descriptive statistics of the three condition groups and the ANOVA results are shown in Tables 1 and 2 . Skewness and kurtosis were within \pm 2 , suggesting that the distributions were normal. Furthermore, effect size measures showed that the proportion of variance explained by the listening proficiency was nil $\left(\omega_{\mathrm{G}}{ }^{2}=.00\right.$; See 2.3 .5 Statistical analyses for details).

Table 1

Summary Statistics of the Three Groups of Listening Proficiency

\begin{tabular}{lcccccc}
\hline & Mean & SD & Skewness & Kurtosis & Minimum & Maximum \\
\hline Multiple choice & 1.15 & 2.10 & 0.77 & 0.40 & -2.04 & 5.88 \\
\hline Open ended & 0.72 & 1.87 & -0.04 & -1.23 & -2.53 & 3.41 \\
\hline Summary cloze & 0.80 & 2.05 & 0.07 & -0.32 & -1.06 & 5.88 \\
\hline
\end{tabular}

Note. The current data $(N=45)$ was combined with data from In'nami (2004; $N=361)$ and analyzed using Facets (Linacre, 2003); Person reliability $=.89$; Infit mean square $=$ 1.00. Descriptive statistics here are based on 45 out of $406(=45+361)$ participants.

Table 2

Analysis of Variance for the Three Groups of Listening Proficiency

\begin{tabular}{lccccccc}
\hline Source & Sum of Squares & $\mathrm{df}$ & Mean Square & $\mathrm{F}$ & Sig. & $\omega_{\mathrm{G}}{ }^{2}$ & Power \\
\hline Task types & 8.83 & 2 & 4.41 & 1.10 & .34 & .00 & .23 \\
\hline Error & 169.32 & 42 & 4.03 & & & & \\
\hline Total & 178.15 & 44 & & & & & \\
\hline
\end{tabular}

\subsubsection{Scoring of the Listening Performance Test}

Although the participants were encouraged to answer in English in the open ended and summary cloze versions, answers in Japanese and those written both in Japanese and in English were also admissible. Answers on the multiple choice task type were dichotomously scored based on a scoring key (UCLES, 2001b). Answers on the open ended and summary cloze task types were also dichotomously scored, using the semantically acceptable word scoring method based on the modified version of the scoring key above. This scoring method is consistent with that used in past studies (Berne, 1992; Wolf, 1991). The agreement rates between the two raters in the open ended and summary cloze items were $99.80 \%$.

\subsubsection{Protocol Data Collection}

Protocol data was collected in a quiet room on an individual basis. The participants were asked to produce retrospective protocols, immediately after they received a 30-second question preview and listened to the listening tape once. Two points are worth discussing here. First, this immediacy was very important to minimize the effects of elaboration and rationalization on the participants' verbal protocols, since 
too large a time lapse between listening to the text and providing verbal protocols constrain the participants' memory. One may consider collecting concurrent verbal protocols (i.e., providing a protocol while listening to the text), but that is not possible in listening studies. Second, in previous studies (Buck, 1990; Wu, 1998) on test-taking processes in listening, a listening text was played twice, and in the case of $\mathrm{Wu}$ was played section by section in the second listening. The listening text was played once, section by section, in Buck. However, listening to the input twice, and section by section enhance listening comprehension by providing more inputs and by limiting the range of possible answers. However, Buck's procedure may be appropriate because one of the research questions examined in that study is listeners' monitoring of their current interpretation of the text. Thus, in the current study, the participants listened to the whole text once.

The participants were asked to tell the researcher, either in Japanese or in English, why they selected or wrote a particular answer each time they solved one item. Before conducting the study, the researcher briefly trained the participants in how to give verbal protocols using similar listening tests. The participants were encouraged to say as much as possible about what they were thinking when they were solving each item. To avoid affecting participants' answers, no leading questions (e.g., 'Did you use information X?' ) were used. However, when the participants were of lower proficiency, only a few protocols were produced (e.g., 'Oh, my, what should I do? I can't understand the text even a bit...') or none at all. The researcher asked whether there was anything they had in mind or what they were thinking at that time. This practice session continued until the participants said that they understood the directions and the researcher ensured this to be so. The participants produced verbal protocols at their own pace. Most of the verbal protocols were in Japanese, except for words and phrases directly quoted from the listening text. All verbal protocols were tape-recorded and transcribed for subsequent analysis.

\subsubsection{Propositional Analysis}

The listening text and features of tasks (i.e., distracters for the multiple choice task, stems for the multiple choice and open ended tasks, and the passage for the summary cloze task) were propositionally analyzed based on Bovair and Kieras (1985), and these propositional lists were used as a template for coding each verbal protocol. Thus, verbal protocol data were attributed to those referring to the features of task types and those referring to the text content. The number of propositions in the features of the tasks was 25 for the multiple choice, 12 for the open ended, and 29 for the summary cloze tasks. The number of propositions in the listening text was 93. All propositional coding of features of tasks, the listening text, and subsequently verbal protocols were conducted by two judges. The agreement rates were $95.58 \%$ for the task features, $80.00 \%$ for the text, and $90.74 \%$ for the verbal protocols. Disagreement was solved 
through discussion. All examples of verbal protocols in the present paper were translated by the researcher from the original Japanese transcriptions. See Appendices for the propositionized Listening Performance Test.

\subsubsection{Statistical Analyses}

The data were analyzed in the following two steps. First, descriptive statistics were calculated, and normality assumptions were inspected. Second, a one-way analysis of variance (ANOVA) was conducted to see whether there were statistical differences in the test scores across the three task types, in the number of propositions referring to the task features, and in the number of propositions referring to the text. Results from a one-way ANOVA were, when necessary, further analyzed by Tukey HSD test.

At the same time, two measures of effect size were calculated. First, generalized omega squared ( $\omega_{\mathrm{G}}{ }^{2}$; Olejnik \& Algina, 2003) was hand-calculated and interpreted. For this, the figure .01 was considered as a small effect, .06 as medium, and .15 as large (Cohen, 1988, pp. 284-288). This measure shows the proportion of variance explained within the range of .00 to 1.00 , and is not influenced by research designs (Olejnik \& Algina). Second, unbiased estimates of $d$ (Hedges \& Olkin, 1985) and its $95 \%$ confidence interval were calculated using Effect Size Calculator and interpreted, considering 10.201 as a small effect, 10.501 as medium, and 10.801 as large (Cohen, pp. 24-27). This measure shows standardized mean differences. One difference between these two measures of effect size is that the former focuses on how much variance in a dependent variable is attributed to variance in one independent variable, whereas the latter focuses on differences between a pair of standardized means.

It is important to note two points here. First, since the data size in the current study ( $N=45$ ). was not large, statistical power was often low (less than .80), and $95 \%$ confidence intervals of $d s$ were large. Thus, especially regarding $d s$, although subsequent discussions were based on $d$ s themselves rather than their confidence intervals, these discussions need to be consolidated using larger datasets. Second, when results of statistical tests and the effect size measures were inconsistent with each other (i.e., statistical differences were not found, but generalized omega squared was large, and vice versa), interpretations were based on effect size measures. This was because effect size measures are less influenced by sample size than statistical tests are (e.g., Kline, 2004). When the effect size measures were interpreted, generalized omega squared was first interpreted. If the calculated generalized omega squared was at least small, $d$ was then interpreted. Throughout the analyses in the present paper, the statistical difference level $(\alpha)$ was set at .05 , and the data were analyzed using SPSS $10.0 \mathrm{E}$.

\section{Results and Discussion}

\subsection{Differences in the Listening Performance Test Scores}


Descriptive statistics are shown in Table 3. Skewness and kurtosis were within \pm 2 , suggesting that the distributions were normal. Reliabilities may be acceptable when we consider the small number of items.

Table 3

Summary Statistics of the Listening Performance Test Scores

\begin{tabular}{lcccccccc}
\hline & Mean & SD & Skewness & Kurtosis & $\begin{array}{c}\text { Min } \\
\text { mum }\end{array}$ & $\begin{array}{c}\text { Maxi- } \\
\text { mum }\end{array}$ & $\begin{array}{c}\text { Full } \\
\text { Mark }\end{array}$ & $\alpha$ \\
\hline Multiple choice & 4.13 & 1.13 & -0.99 & -0.40 & 2 & 5 & 5 & .60 \\
\hline Open ended & 3.00 & 1.25 & -0.25 & -1.19 & 1 & 5 & 5 & .58 \\
\hline Summary cloze & 3.13 & 1.46 & -1.06 & 0.03 & 0 & 5 & 5 & .71 \\
\hline
\end{tabular}

Note. $\alpha=$ Cronbach's $\alpha$.

A one-way ANOVA was conducted to see whether there were statistical differences in the test scores in the Listening Performance Test across the three task types. Results appeared in Tables 4 and 5 and showed that there were statistical differences between the task types, $F(2,42)=3.69, p=.03$. Results of Tukey HSD test showed that there were statistical differences in the test scores between the multiple choice and open ended task types. Furthermore, effect size measures showed that the proportion of variance explained by the task types was medium $\left(\omega_{\mathrm{G}}{ }^{2}=.11\right)$, and that the score differences were large between the multiple choice and open ended tasks $(d=0.92$ [95\% confidence interval $=0.17$ to 1.68$]$ ), medium between the multiple choice and summary cloze tasks ( $d=0.75$ [0.01 to 1.49$]$ ), and none between the open ended and summary cloze tasks ( $d=-0.09[-0.81$ to 0.62$])$.

Table 4

Analysis of Variance for the Listening Performance Test Scores

\begin{tabular}{lccccccc}
\hline Source & Sum of Squares & df & Mean Square & F & Sig. & $\omega_{\mathrm{G}}{ }^{2}$ & Power \\
\hline Task types & 11.51 & 2 & 5.76 & 3.69 & .03 & .11 & .65 \\
\hline Error & 65.47 & 42 & 1.56 & & & & \\
\hline Total & 76.98 & 44 & & & & & \\
\hline
\end{tabular}

Table 5

Tukey HSD Test for the Listening Performance Test Scores

\begin{tabular}{llccccc}
\hline & $\begin{array}{c}\text { Mean difference } \\
\text { (I-J) }\end{array}$ & $\begin{array}{c}\text { Std. } \\
\text { Error }\end{array}$ & $\begin{array}{c}\text { Sig } \\
\text {. }\end{array}$ & \multicolumn{2}{c}{$\begin{array}{c}95 \% \text { Confidence } \\
\text { Interval }\end{array}$} \\
\hline (I) task types & (J) task types & & & & $\begin{array}{c}\text { Lower } \\
\text { Bound }\end{array}$ & $\begin{array}{c}\text { Upper } \\
\text { Bound }\end{array}$ \\
& & & & & \\
& & 1.13 & 0.46 & .04 & 0.02 & 2.24 \\
\hline Multiple choice & Open ended & 1.00 & 0.46 & .08 & -0.11 & 2.11 \\
\hline & Summary cloze & 1.00 & 0.46 & .95 & -1.24 & 0.97 \\
\hline Open ended & Summary cloze & -0.13 & & & &
\end{tabular}


Further analysis was conducted to see which item was responsible for the difficulty across the three tasks. Among the 5 items, item 2 had an extremely large score gap between the tasks. Since descriptive statistics in Table 6 showed that the data distribution was not normal, neither one-way ANOVA nor effect size measures were appropriate. Nevertheless, the descriptive statistics themselves seemed to indicate that the multiple choice task was easier than the open ended and summary cloze tasks, and that the open ended and summary cloze tasks were of the same difficulty.

Table 6

Summary Statistics of the Listening Performance Test Scores Item 2

\begin{tabular}{lccccccc}
\hline & Mean & SD & Skewness & Kurtosis & $\begin{array}{c}\text { Mini- } \\
\text { mum }\end{array}$ & $\begin{array}{c}\text { Maxi- } \\
\text { mum }\end{array}$ & $\begin{array}{c}\text { Full } \\
\text { Mark }\end{array}$ \\
\hline Multiple choice & .67 & .49 & -0.79 & -1.62 & 0 & 1 & 1 \\
\hline Open ended & .13 & .35 & 2.41 & 4.35 & 0 & 1 & 1 \\
\hline Summary cloze & .13 & .36 & 2.41 & 4.35 & 0 & 1 & 1 \\
\hline
\end{tabular}

In item 2, many participants in the open ended and summary cloze tasks failed to listen the word "gloves" correctly. Their main incorrect responses were "glasses" and "drugs." These responses suggested that the participants did not differentiate phonemes as in gloves, glasses, and drugs, or that they recognized the word glove but failed to comprehend the conversation. Since the participants were randomly assigned to one of the three task types, those taking the multiple choice task must have also had the same problem. However, the multiple choice task had options, and thus the participants taking the multiple choice task took advantage of these options.

The order of task difficulty found in the current study is consistent with Berne (1992) and Wolf (1991). Although these two studies use listening tests with low reliabilities (KR 21 calculated by the current researcher $=.37$ and .36 for the multiple choice, .53 and .59 for the open ended, and .38 and .49 for the summary cloze tasks in Berne and Wolf, respectively), the task difficulty is consistent and thus more generalizable across context. Possible reasons for this task difficulty are that the multiple choice task is easier because options serve as clues to the correct answer, and that the open ended and summary cloze tasks are more difficult since they do not have such options. It is also important to note that the equal difficulty of the open ended and summary cloze tasks may be due to their parallel task design. Both tasks are very similarly designed except that the open ended task uses an interrogative form and that the summary cloze task uses a declarative form. This suggests that task difficulty may be equal as long as the two task types are closely designed and minimal changes are added.

\subsection{Differences in Propositions Used Referring to the Task Features}


Descriptive statistics are shown in Table 7. Skewness and kurtosis were within \pm 2 , suggesting that the distributions were normal.

Table 7

Summary Statistics of the Number of Propositions Used Referring to the Task Features

\begin{tabular}{lccccccc}
\hline & Mean & SD & Skewness & Kurtosis & Min & Max & Total \\
\hline Multiple choice & 9.20 & 4.78 & -0.70 & -0.78 & 0 & 15 & 25 \\
\hline Open ended & 6.80 & 4.93 & -0.54 & -1.59 & 0 & 12 & 12 \\
\hline Summary cloze & 9.67 & 8.79 & 1.05 & -0.07 & 0 & 27 & 29 \\
\hline
\end{tabular}

Note . Min $=$ Minimum; Max = Maximum; Total = Total number of the propositions in the task features.

A one-way ANOVA was conducted to see whether there were statistical differences in the number of propositions used that were related to task types. Results appeared in Table 8 and showed that there were no statistical differences between the task types, $F(2,42)=0.86, p=.43$. Furthermore, effect size measures showed that the proportion of variance explained by the task types was nil $\left(\omega_{G}{ }^{2}=.00\right)$. This suggests that the participants used the same number of propositions referring to the task types when they answered the test items across the three task types.

Table 8

Analysis of Variance for the Number of Propositions Used Referring to the Task Features

\begin{tabular}{lccccccc}
\hline Source & Sum of Squares & $\mathrm{df}$ & Mean Square & $\mathrm{F}$ & Sig. & $\omega_{\mathrm{G}}{ }^{2}$ & Power \\
\hline Task types & 70.98 & 2 & 35.49 & 0.86 & .43 & .00 & .19 \\
\hline Error & 1742.13 & 42 & 41.48 & & & & \\
\hline Total & 1813.11 & 44 & & & & & \\
\hline
\end{tabular}

3.3 Differences in Propositions Used Referring to the Text Features

\subsubsection{Overall Results}

Descriptive statistics are shown in Table 9. Skewness and kurtosis were within \pm 2 , suggesting that the distributions were normal.

Table 9

Summary Statistics of the Number of Propositions Used Referring to the Text

\begin{tabular}{lccccccc}
\hline & Mean & SD & Skewness & Kurtosis & Min & Max & Total \\
\hline Multiple choice & 28.93 & 8.66 & -0.59 & -0.40 & 11 & 42 & 93 \\
\hline Open ended & 22.27 & 11.44 & 0.29 & -0.64 & 4 & 40 & 93 \\
\hline Summary cloze & 20.33 & 9.24 & -0.23 & -0.25 & 3 & 35 & 93 \\
\hline
\end{tabular}

Note . Min $=$ Minimum; $\operatorname{Max}=$ Maximum; Total $=$ Total number of the propositions in the text. 
A one-way ANOVA was conducted to see whether there were statistical differences in the number of propositions used that were related to the text. Results appeared in Table 10 and showed that there were no statistical differences between the task types, $F(2,42)=3.15, p=.05$. However, effect size measures showed that the proportion of variance explained by the task types was medium $\left(\omega_{\mathrm{G}}{ }^{2}=.09\right)$, and that the differences in the number of propositions were medium between the multiple choice and open ended tasks ( $d=0.64$ [-0.10 to 1.37]), large between the multiple choice and summary cloze tasks ( $d=0.93$ [0.18 to 1.69$]$ ), and none between the open ended and summary cloze tasks $(d=0.18[-0.54$ to 0.90$])$.

Table 10

Analysis of Variance for the Number of Propositions Used Referring to the Text

\begin{tabular}{lccccccc}
\hline Source & Sum of Squares & df & Mean Square & F & Sig. & $\omega_{\mathrm{G}}{ }^{2}$ & Power \\
\hline Task types & 610.71 & 2 & 305.36 & 3.15 & .05 & .09 & .57 \\
\hline Error & 4075.20 & 42 & 97.03 & & & & \\
\hline Total & 4685.91 & 44 & & & & & \\
\hline
\end{tabular}

The results seem to show that since the multiple choice task required the participants to listen to the options and differentiate the answer key from the distractors, the number of the propositions used attributed to the text in the multiple choice task was larger than that of the open ended and summary cloze tasks. Although the participants used propositions most in the multiple choice and least in the summary cloze tasks, when the degree of the propositions used was compared with the total number of the propositions in the text, another picture emerged. Even in the multiple choice, only about $30 \%$ of the propositions in the text were used for answering the test items. In the open ended and summary cloze tasks, only about $20 \%$ of the propositions in the text were used. These results suggested that the participants used a relatively small number of the propositions related to the text when answering the test items. There are two possible reasons for the low frequency of use of the propositions. First, the participants' test-taking processes must have been rather automatized and they were not fully elicited by verbal protocol methods. This is quite likely because the test scores in Table 3 are not so low and thus the participants must have processed some text information to reach the correct answers. Second, since collecting concurrent verbal protocols is not possible in listening studies, there is always a time lag between listening to the tape and providing verbal protocols. This suggests that the participants must have used more propositions but failed to produce them in the immediate verbal protocols.

\subsubsection{Item-Based Analysis}

Descriptive statistics are shown in Table 11. Skewness and kurtosis were within 
\pm 2 , suggesting that the distributions were normal.

Table 11

Item Summary Statistics of the Number of Propositions Used Referring to the Text

\begin{tabular}{lccccccc}
\hline & Mean & SD & Skewness & Kurtosis & Min & Max & Total \\
\hline Multiple choice 1 & 8.40 & 2.77 & -1.04 & 0.57 & 2 & 11 & 19 \\
\hline Open ended 1 & 6.00 & 4.02 & 0.62 & -0.87 & 1 & 14 & 19 \\
\hline Summary cloze 1 & 8.93 & 4.23 & -0.57 & 0.83 & 0 & 17 & 19 \\
\hline Multiple choice 2 & 5.00 & 2.70 & -0.28 & -1.60 & 1 & 8 & 14 \\
\hline Open ended 2 & 2.67 & 2.09 & 0.24 & -1.17 & 0 & 6 & 14 \\
\hline Summary cloze 2 & 3.33 & 2.16 & 0.48 & -0.05 & 0 & 8 & 14 \\
\hline Multiple choice 3 & 3.07 & 1.75 & 1.08 & 0.57 & 1 & 7 & 8 \\
\hline Open ended 3 & 1.93 & 2.15 & 0.89 & -0.36 & 0 & 6 & 8 \\
\hline Summary cloze 3 & 1.93 & 1.58 & 0.13 & -1.45 & 0 & 4 & 8 \\
\hline Multiple choice 4 & 6.27 & 1.79 & 0.66 & -0.29 & 4 & 10 & 11 \\
\hline Open ended 4 & 5.40 & 1.88 & 0.44 & -0.13 & 2 & 9 & 11 \\
\hline Summary cloze 4 & 5.67 & 2.77 & 0.40 & -0.44 & 2 & 11 & 11 \\
\hline Multiple choice 5 & 6.20 & 2.51 & -1.01 & 1.34 & .0 & 9 & 26 \\
\hline Open ended 5 & 6.80 & 4.07 & -0.09 & -1.06 & 1 & 14 & 26 \\
\hline Summary cloze 5 & 5.93 & 4.10 & -0.24 & -1.58 & 0 & 11 & 26 \\
\hline Note Mulp cho
\end{tabular}

Note. Multiple choice $1=$ item 1 in the multiple choice task. Min = Minimum; Max $=$ Maximum.

A one-way ANOVA was conducted to see how the propositions referring to the text were used with each item. Selected results appeared in Tables 12 and 13 and showed that there were statistical differences between the task types in item $2, F(2,42)$ $=8.78, p=.00$. Results of Tukey HSD test showed that there were statistical differences in the number of the propositions used that were related to the text between the multiple choice and open ended tasks, and between the multiple choice and summary cloze tasks. Effect size measures showed that the proportion of variance explained by the task types was nil to large $\left(\omega_{\mathrm{G}}{ }^{2}=.05, .26, .04, .03\right.$, and .00 for each item), and that the differences in the number of propositions were nil to large in items 1,2 , and 3 , and nil to medium in item 4, as shown in Table 14.

Table 12

Analysis of Variance for the Number of Propositions Used Referring to the Text: Item 2

\begin{tabular}{|c|c|c|c|c|c|c|c|}
\hline Source & Sum of Squares & $\mathrm{df}$ & Mean Square & $\bar{F}$ & Sig. & $\omega_{G}^{2}$ & Power \\
\hline Task types & 2.84 & 2 & 1.42 & 8.78 & .00 & .26 & .96 \\
\hline Error & 6.80 & 42 & .16 & & & & \\
\hline Total & 9.64 & 44 & & & & & \\
\hline
\end{tabular}


Table 13

Tukey HSD Test for the Number of Propositions Used Referring to the Text: Item 2

\begin{tabular}{|c|c|c|c|c|c|c|}
\hline & & $\begin{array}{c}\text { Mean difference } \\
(I-J)\end{array}$ & $\begin{array}{l}\text { Std. } \\
\text { Error }\end{array}$ & Sig. & $\begin{array}{r}95 \% \mathrm{C} \\
\text { Int }\end{array}$ & $\begin{array}{l}\text { nfidence } \\
\text { rval }\end{array}$ \\
\hline (I) task types & $(\mathrm{J})$ task types & & & & $\begin{array}{l}\text { Lower } \\
\text { Bound }\end{array}$ & $\begin{array}{l}\text { Upper } \\
\text { Bound }\end{array}$ \\
\hline \multirow[t]{2}{*}{ Multiple choice } & Open ended & .53 & .15 & .00 & .18 & .89 \\
\hline & Summary cloze & .53 & .15 & .00 & .18 & .89 \\
\hline Open ended & Summary cloze & .00 & .15 & 1.00 & -.36 & .36 \\
\hline
\end{tabular}

Table 14

Effect Size (d) of the Number of Propositions Used Referring to the Text

\begin{tabular}{cccccc}
\hline MC1-OE1 & MC1-SC1 & OE1-SC1 & MC2-OE2 & MC2-SC2 & OE2-SC2 \\
\hline $\begin{array}{c}\text { O.68 [-0.06 } \\
\text { to 1.41] }\end{array}$ & $\begin{array}{c}-0.14[-0.86 \\
\text { to } 0.57]\end{array}$ & $\begin{array}{c}-0.69[-1.43 \\
\text { to } 0.05]\end{array}$ & $\begin{array}{c}0.94[0.18 \text { to } \\
1.69]\end{array}$ & $\begin{array}{c}0.66[-0.07 \\
\text { to } 1.40]\end{array}$ & $\begin{array}{c}-0.30[-1.02 \\
\text { to 0.42] }\end{array}$ \\
\hline MC3-OE3 & MC3-SC3 & OE3-SC3 & MC4-OE4 & MC4-SC4 & OE4-SC4 \\
\hline $0.57[-0.16$ & $0.67[-0.07$ & $0.00[-0.72$ & $0.46[-0.26$ & $0.25[-0.47$ & $-0.11[-0.83$ \\
to 1.30$]$ & to 1.40$]$ & to 0.72] & to 1.19] & to 0.97] & to 0.61] \\
\hline
\end{tabular}

Note. $\mathrm{MC} 1$ = item 1 in the multiple choice task; OE1 = item 1 in the open ended task; $\mathrm{SCl}=$ item 1 in the summary cloze task.

Further analysis was conducted to investigate which propositions were used in answering the test items. Results are presented in Figures 1 to 5 . The $\mathrm{x}$-axis refers to the proposition number labels, and the $y$-axis refers to the number of the test takers using those proposition labels. Consistent with the results of the effect size measures, there were more differences in the number of the propositions in items 1 to 3 than items 4 and 5. Items 1 to 3 are shown in Table 15, and items 4 and 5 are shown in Table 16 below.

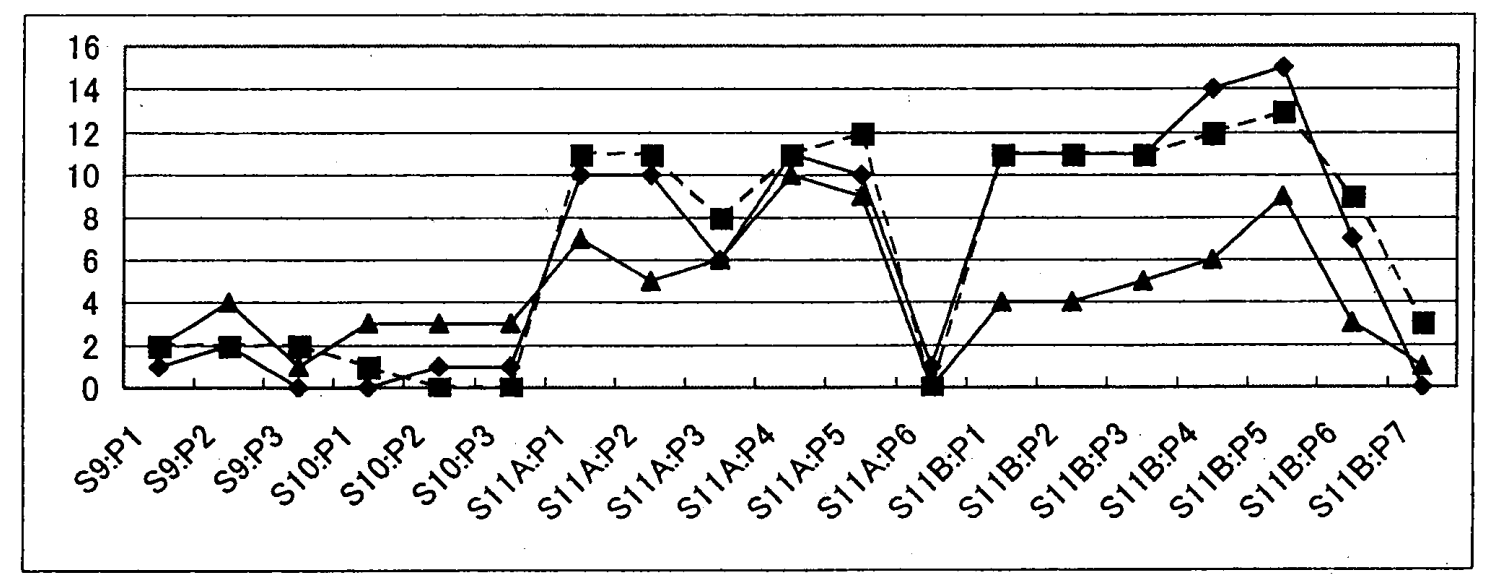

Figure 1. The number of propositions used for referring to the text in item 1 Note. $\boldsymbol{\nabla}=$ multiple choice tasks; $\boldsymbol{\Delta}$ = open ended tasks; $\boldsymbol{\square}=$ summary cloze tasks. These indicators also apply to Figures 2-5. 


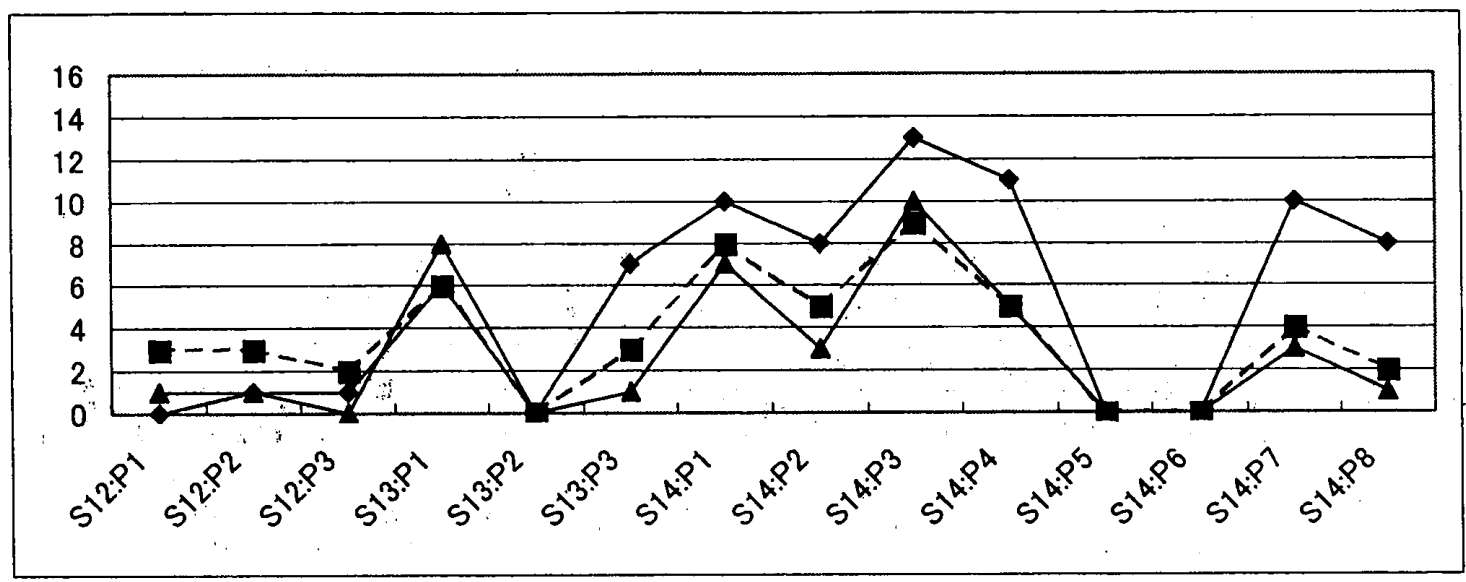

Figure 2. The number of propositions used for referring to the text in item 2

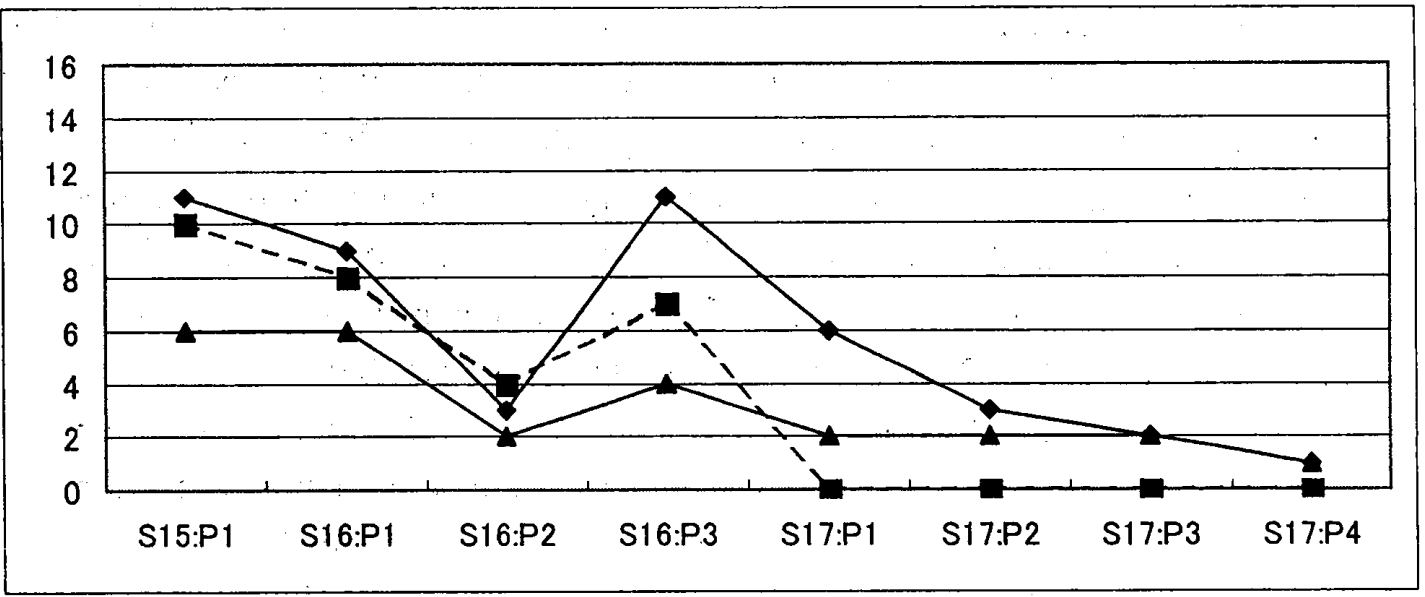

Figure 3. The number of propositions used for referring to the text in item 3

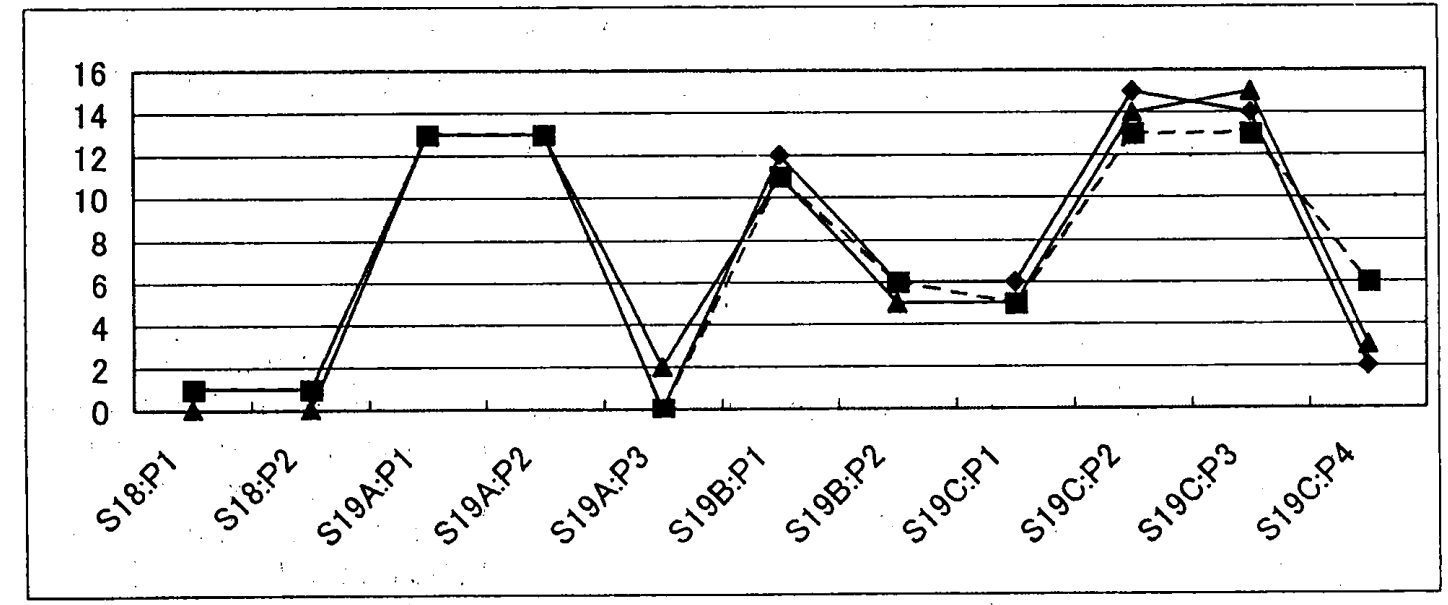

Figure 4 . The number of propositions used for referring to the text in item 4 


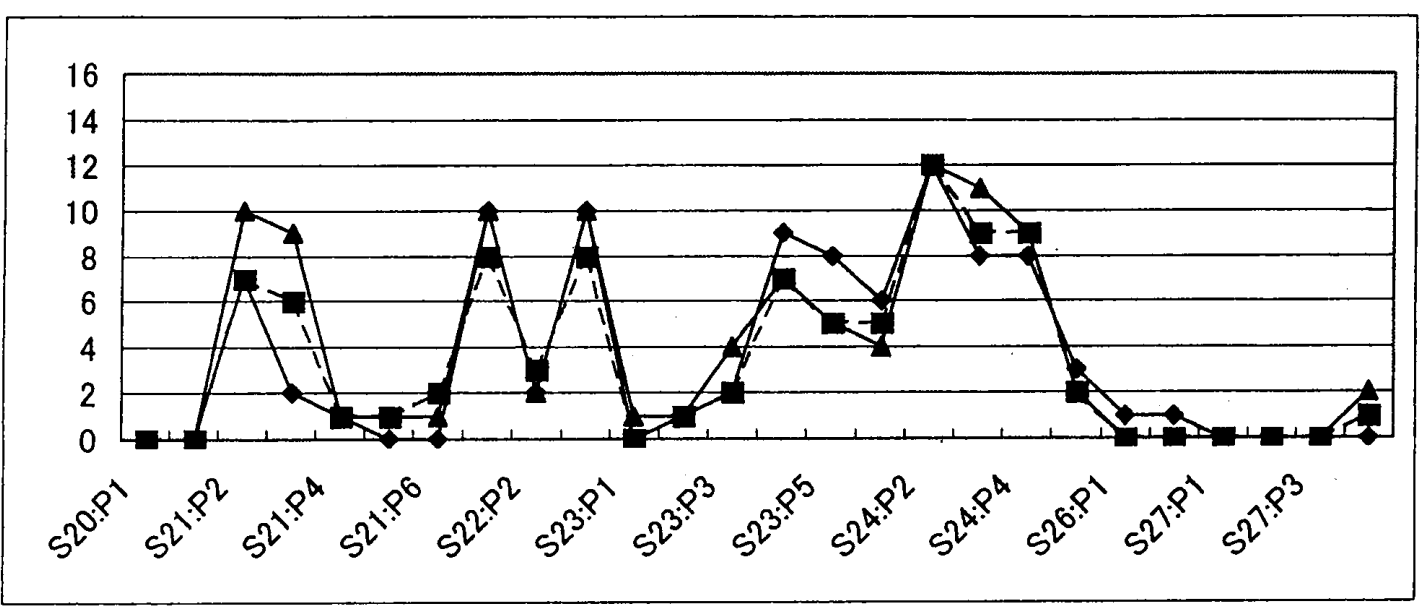

Figure 5. The number of propositions used for referring to the text in item 5

Two reasons may explain these differences. First, one of the characteristics shared by items 1 and 3 was that these items turned out to be constructed in a less parallel way, although every effort was made to make them as parallel as possible. This was especially true of item 1, in which only the summary cloze task had an introduction section. Although the introduction could be deleted, doing so may have caused the characteristics of the summary cloze task to be lost and may have made the task closer to the open ended task. Item 3, on the other hand, was more parallel across the three task types than item 1 was. However, the acceptable range of the answers in item 3 in the open ended and summary cloze tasks was wider than the other items. In fact, in addition to "old," other answers "not expensive" and "not big" were also acceptable. Thus, once the participants reached one of these answers, especially in the open ended and summary cloze tasks, they stopped searching for other possible answers. Second, item 2 was the most difficult item, especially in the open ended and summary cloze tasks, as shown in Table 6 and discussed above. Item 2 was also the one in which generalized omega squared and $d$ are large. Thus, the item difficulty and the number of the propositions used that were related to the text were connected.

Table 15

Items 1 to 3

\begin{tabular}{|c|c|c|}
\hline Multiple choice & Open ended & Summary cloze \\
\hline $\begin{array}{l}\text { 1. How much money was } \\
\text { in the bag? } \\
\text { (A) £20* } \\
\text { (B) £40 } \\
\text { (C) £50 }\end{array}$ & $\begin{array}{l}\text { 1. How much money was } \\
\text { in the bag? } \\
£ \quad 20\end{array}$ & $\begin{array}{l}\text { A woman lost her bag and } \\
\text { reported it to the police. } \\
\text { She did not have much } \\
\text { money in her bag. In fact, } \\
\text { she had only } £([1] \\
\text { twenty } / 20) \text { in the bag. }\end{array}$ \\
\hline $\begin{array}{l}\text { 2. What else was in the } \\
\text { bag? } \\
\text { (A) credit card } \\
\text { (B) driving license }\end{array}$ & $\begin{array}{l}2 . \text { What else was in the } \\
\text { bag? } \\
\text { gloves }\end{array}$ & $\begin{array}{l}\text { She also had ([2] gloves) } \\
\text { in the bag. }\end{array}$ \\
\hline
\end{tabular}




\begin{tabular}{l|l|l}
\hline (C) gloves* & & \\
\hline 3. The bag was & 3. How was the bag? & The bag she lost was ([3] \\
(A) old.* & old). \\
(B) expensive. & old & \\
(C) big. &
\end{tabular}

Note. *answer keys in the multiple choice version. Boldfaced letters in the open ended and summary cloze versions indicate examples of correct answers. These notes also apply to Table 16.

In contrast to items 1 to 3 , the participants used more or less the same number of the propositions related to the listening text in items 4 and 5. Overall, these two items, as shown in Table 16 below, looked parallel across the three tasks. Although the summary cloze task in item 5 was less parallel to the multiple choice and open ended tasks since it contained more information (i.e., to tell whether he was able to find her bag), this information might have provided less clues to answers than that from the introduction section in item 1 .

Table 16

Items 4 and 5

\begin{tabular}{|c|c|c|}
\hline Multiple choice & Open ended & Summary cloze \\
\hline $\begin{array}{l}\text { 4. What time did the } \\
\text { woman lose the bag? } \\
\text { (A) } 9.30 \\
\text { (B) } 10.00^{*} \\
\text { (C) } 10.30\end{array}$ & $\begin{array}{l}\text { 4. What time did the } \\
\text { woman lose the bag? } \\
10.00\end{array}$ & $\begin{array}{l}\text { The time she lost her bag } \\
\text { was at }([4] 10.00) \text {. }\end{array}$ \\
\hline $\begin{array}{l}\text { 5. The policeman will } \\
\text { telephone her in the } \\
\text { (A) morning.* } \\
\text { (B) afternoon. } \\
\text { (C) evening. }\end{array}$ & $\begin{array}{l}\text { 5. When will the policeman } \\
\text { telephone her? } \\
\text { (in the) morning }\end{array}$ & $\begin{array}{l}\text { The policeman will call her } \\
\text { in the ([5] morning) to tell } \\
\text { whether he was able to find } \\
\text { her bag. }\end{array}$ \\
\hline
\end{tabular}

Thus, based on the results of the item-based analysis, it may be reasonable to say that the more task types were parallel, the more test-takers used similar propositions related to texts when answering test items. This finding supports the meta-analytic result of Rodriguez (2003), which compares the trait equivalence of multiple choice and open ended tasks and finds that when the two task types are constructed using the same stem, correlations between them approach unity. The finding of the current study also concurs with Fulcher (1996), which argues for the generalizability of test performance measures.

\section{Conclusion}

The current study has examined (a) whether test scores are different across the three task types (i.e., multiple choice, open ended, and summary cloze tasks), (b) whether the number of propositions used that are related to the task types in answering test items is different across the three task types, and (c) whether the number of 
propositions used that are related to the listening text in answering test items is different across the three task types. First, multiple choice tasks are easier than open ended and summary cloze tasks, becaüse multiple choice tasks have options and thus test-takers can take advantage of these options. It is also important to note that the equal difficulty of the open ended and summary cloze tasks may be due to their parallel task design. Thus, task difficulty may be equal as long as the two task types are closely designed and minimal changes are added. Second, test-takers use the same number of propositions referring to task types when answering test items across multiple choice, open ended, and summary cloze tasks. Third, the number of propositions used that are related to texts is the largest in the multiple choice task, followed by the open ended and summary cloze tasks. Item analysis shows that the more task types are parallel, the more test-takers use similar propositions related to texts when answering test items. This finding is very important in terms of the generalizability of test performance.

Finally, future research is necessary to see how the propositional use by test-takers is related to their test scores, and to see whether and how the effects of task types vary across listening proficiency levels.

\section{Acknowledgements}

This research was supported by a grant from the Japan Language Testing Association. I am deeply indebted to Akihiko Mochizuki, Yuji Ushiro, and Rie Koizumi for their useful comments.

\section{References}

Alderson, J. C. (1990). Testing reading comprehension skills (Part two): Getting students to talk about taking a reading test (A pilot study). Reading in a Foreign Language, 7, 465-503.

Berne, J. E. (1992). The effects of text type, assessment task, and target language experience on foreign language learners' performance on listening comprehension tests. (UMI No. 9236396)

Bovair, S., \& Kieras, D. E. (1985). A guide to propositional analysis for research on technical prose. In B. K. Britton \& J. B. Black (Eds.), Understanding expository text: A theoretical and practical handbook for analyzing explanatory text (pp. 315-362). Hillsdale, NJ: Erlabum.

Brindley, G. (1998). Outcome-based assessment and reporting in language learning programmes: A review of the issues. Language Testing, 15, 45-85.

Buck, G. (1990). The testing of second language listening comprehension. Unpublished doctoral dissertation, University of Lancaster.

Buck, G. (1991). The testing of listening comprehension: An introspective study. Language Testing, 8, 67-91

Buck, G. (2001). Assessing listening. Cambridge University Press. 
Carrell, P. L. (1985). Facilitating ESL reading by teaching text structure. TESOL Quarterly, 19, 727-752.

Chapelle, C. A. (1998). Construct definition and validity inquiry in SLA research. In L. F. Bachman \& A. D. Cohen (Eds.), Interfaces between second language acquisition and language testing research (pp. 32-70). Cambridge University Press.

Chapelle, C. A. (1999). Validity in language assessment. Annual Review of Applied Linguistics, 19, 254-272.

Cohen, J. (1988). Statistical power analysis for the behavioral sciences (2nd ed). Hillsdale, NJ: Erlbaum.

Effect Size Calculator [Computer software]. Retrieved from http://www.cemcentre.org/ ebeuk/research/effectsize/EffectSizeCalculator.xls

Fulcher, G. (1996). Testing tasks: Issues in task design and the group oral. Language Testing, 13, 23-51.

Hedges, L. V., \& Olkin, I. (1985). Statistical methods for meta-analysis. New York: Academic Press.

In'nami, Y. (2001). The effects of text and task on the listening scores of Japanese university students. JLTA Journal, 4, 56-75.

In'nami, Y. (2004). The effects of listening proficiency and task on the listening test performance of Japanese EFL learners. Unpublished master's thesis, University of Tsukuba, Japan.

Kintsch, W. (1998). Comprehension: A paradigm for cognition. Cambridge University Press.

Kline, R. B. (2004). Beyond significance testing: Reforming data analysis methods in behavioral research. Washington, DC: American Psychological Association.

Kobayashi, M. (1995). Effects of text organization and test format on reading comprehension test performance. Unpublished doctoral dissertation, Thames Valley University, London.

Kroll, B. (1977). Combining ideas in written and spoken English: A look at subordination and coordination. In E. O. Keenan \& T. L. Bennett (Eds.), Southern California Occasional Papers in Linguistics No. 5: Discourse across time and space (pp. 69-108). Los Angeles: Department of Linguistics, University of Southern California.

Kunnan, A. J. (2000). Fairness and justice for all. In A. J. Kunnan (Ed.), Fairness and validation in language assessment: Selected papers from the 19th Language Testing Research Colloquium, Orland, Florida (pp. 1-14). Cambridge University Press.

Linacre, J. M. (2003). Facets: Rasch-measurement computer program (Version 3.45.2) [Computer software]. Chicago: MESA Press.

McKay, P. (2000). On ESL standards for school-age learners. Language Testing, 17, 
185-214.

Messick, S. (1996). Validity and washback in language testing. Language Testing, 13, 241-256.

Olejnik, S., \& Algina, J. (2003). Generalized eta and omega squared statistics: Measures of effect size for some common research designs. Psychological Methods, 8 , 434-447.

Riley, G. L., \& Lee, J. F. (1996). A comparison of recall and summary protocols as measures of second language reading comprehension. Language Testing; 13 , 173-189.

Rodriguez, M. C. (2003). Construct equivalence of multiple-choice and constructedresponse items: A random effects synthesis of correlations. Journal of Educational Measurement, 40, 163-184.

Ross, S. (1997). An introspective analysis of listener inferencing on a second language listening task. In G. Kasper \& E. Kellerman (Eds.), Communication strategies: Psycholinguistic and sociolinguistic perspectives (pp. 216-237). New York: Longman.

Shohamy, E. (1984). Does the testing method make a difference? The case of reading comprehension. Language Testing, 1, 147-170.

Storey, P. (1997). Examining the test-taking process: A cognitive perspective on the discourse cloze test. Language Testing, 14, 214-231.

University of Cambridge Local Examinations Syndicate. (2001a). Cambridge KET handbook. Retrieved December 10, 2003, from http://www.cambridge-efl.org/support/dloads/ket/ket_hb_intro_03.pdf

University of Cambridge Local Examinations Syndicate. (2001b). Cambridge Key English Test 2 with answers. Cambridge University Press.

University of Cambridge Local Examinations Syndicate. (2001c). Cambridge Key English Test 2 (Cassette Recording). Cambridge University Press.

Wolf, D. F. (1991). The effects of task, language of assessment, and target language experience on foreign language learners performance on reading comprehension tests. (UMI No. 9124507)

$\mathrm{Wu}, \mathrm{Y}$. (1998). What do tests of listening comprehension test? A retrospection study of EFL test-takers performing a multiple-choice task. Language Testing, 15, 2144.

Yamashita, J. (2003). Processes of taking a gap-filling test: Comparison of skilled and less skilled EFL readers. Language Testing, 20, 267-293.

Appendices
Appendix 1 Propositionized Listening Text

Male: Good morning, madam. Can I help you?

S1:P1 (MOD MORNING GOOD) / S1:P2 (MOD P1 MADAM)

S2:P1 (ABLE-TO WOMAN P2) / S2:P2 (HELP MAN WOMAN) 
Female: Yes. I've lost my bag.

S3:P1 (YES, S2:P1)

S4:P1 (LOSE WOMAN BAG) / S4:P2 (POSSESS WOMAN BAG)

Male: Oh, I am sorry. Now, where did you lose it?

S5:P1 (MOD WOMAN SORRY)

S6:P1 (WHERE P2) / S6:P2 (LOSE WOMAN BAG)

Female: In the town center. I had it when I got off the bus.

S7:P1 (IN S6:P2 CENTER) / S7:P2 (MOD CENTER TOWN)

S8:P1 (WHEN P2 P3) / S8:P2 (POSSESS WOMAN BAG) / S8:P3 (GET-OFF

WOMAN BUS)

Male: Was there much money in the bag?

S9:P1 (EXIST MONEY) / S9:P2 (AMOUNT-OF MONEY MUCH) / S9:P3 (IN P1 BAG)

Female: No, there wasn't. I usually have forty or fifty pounds in it, but today I think there was only about twenty.

S10:P1 (NO) / S10:P2 (EXIST S9:P2) / S10:P3 (NEGATE P2)

S11A:P1 (POSSESS WOMAN POUND) / S11A:P2 (MOD P1 USUAL) / S11A:P3

(OR P4 P5) / S11A:P4 (NUMBER-OF POUND FORTY) / S11A:P5 (NUMBER-OF POUND FIFTY) / S11A:P6 (IN P1 BAG)

S11B:P1 (BUT S11A:P1 S11B:P2) / S11B:P2 (THINK WOMAN P4) / S11B:P3 (TIME

P4 TODAY) / S11B:P4 (EXIST POUND) / S11B:P5 (NUMBER-OF POUND

TWENTY) / S11B:P6 (MOD TWENTY ONLY) / S11B:P7 (MOD TWENTY ABOUT)

Male: What else was in the bag?

S12:P1 (WHAT-ELSE P2) / S12:P2 (EXIST \$) / S12:P3 (IN P2 BAG)

Female: Just my gloves. I left my credit card and driving license at home.

S13:P1 (EXIST GLOVE) / S13:P2 (POSSESS WOMAN GLOVE) / S13:P3 (MOD GLOVE JUST)

S14:P1 (LEAVE WOMAN CARD) / S14:P2 (LEAVE WOMAN LICENSE) / S14:P3

(MOD CARD CREDIT) / S14:P4 (MOD LICENSE DRIVING) / S14:P5 (POSSESS

WOMAN CARD) / S14:P6 (POSSESS WOMAN LICENSE) / S14:P7 (AT P1 HOME) S14:P8 (AT P2 HOME)

Male: Was the bag expensive?

S15:P1 (MOD BAG EXPENSIVE)

Female: No, it was an old one. It wasn't big enough really.

S16:P1 (NEGATE S15:P1) / S16:P2 (ISA BAG1 BAG2) / S16:P3 (MOD BAG2 OLD)

S17:P1 (MOD BAG1 BIG) / S17:P2 (NEGATE P1) / S17:P3 (MOD BIG ENOUGH)

S17:P4 (MOD P2 REALLY)

Male: Now, what time did you lose it?

S18:P1 (WHAT-TME P2) / S18:P2 (LOSE WOMAN BAG)

Female: Well, I left home about nine thirty and the bus takes half an hour, so I lost it about ten o'clock.

S19A:P1 (LEAVE WOMAN HOME) / S19A:P2 (TIME P1 NINE-THIRTY) / S19A:P3 (MOD P2 ABOUT)

S19B:P1 (TAKE BUS HALF-AN-HOUR) / S19B:P2 (AND S19A:P1 S19B:P1)

S19C:P1 (SO S19C:P2 S19B:P2) / S19C:P2 (LOSE WOMAN BAG) / S19C:P3 (TIME 


\section{P2 TEN O'CLOCK) / S19C:P4 (MOD P3 ABOUT)}

Male: Right. Well, I'll phone you tomorrow to tell you if we find it. Are you at home in the afternoon?

S20:P1 (RIGHT)

S21:P1 (IN-ORDER-TO P2 P4) / S21:P2 (PHONE MAN WOMAN) / S21:P3 (TIME P2 TOMORROW) / S21:P4 (TELL MAN WOMAN P5) / S21:P5 (IF P6) / S21:P6 (FIND MAN BAG)

S22:P1 (EXIST WOMAN) / S22:P2 (AT P1 HOME) / S22:P3 (IN P1 AFTERNOON)

Female: Sorry, I'm going out until the evening. Could you phone before ten in the morning?

S23:P1 (SORRY) / S23:P2 (NEGATE S22:P2) / S23:P3 (NEGATE S22:P3) / S23:P4

(GO-OUT WOMAN) / S23:P5 (UNTL P4 EVENING)

S24:P1 (ABLE-TO MAN P2) / S24:P2 (PHONE MAN) / S24:P3 (BEFORE P2 TEN)

S24:P4 (IN P2 MORNING)

Male: Certainly. I'll call then. Now, what's your number?

S25:P1 (CERTAIN)

S26:P1 (CALL MAN) / S26:P2 (TIME P1 THEN)

S27:P1 (WHAT P2) / S27:P2 (ISA NUMBER \$) / S27:P3 (POSSESS WOMAN

NUMBER)

Female: It's oh three six two...

S28:P1 (REF NUMBER OH-THREE-SIX-TWO)

Note $. \mathrm{S}=$ Sentence $\mathrm{P}=$ Proposition.

Appendix 2 A Sample of Propositionized Listening Tasks

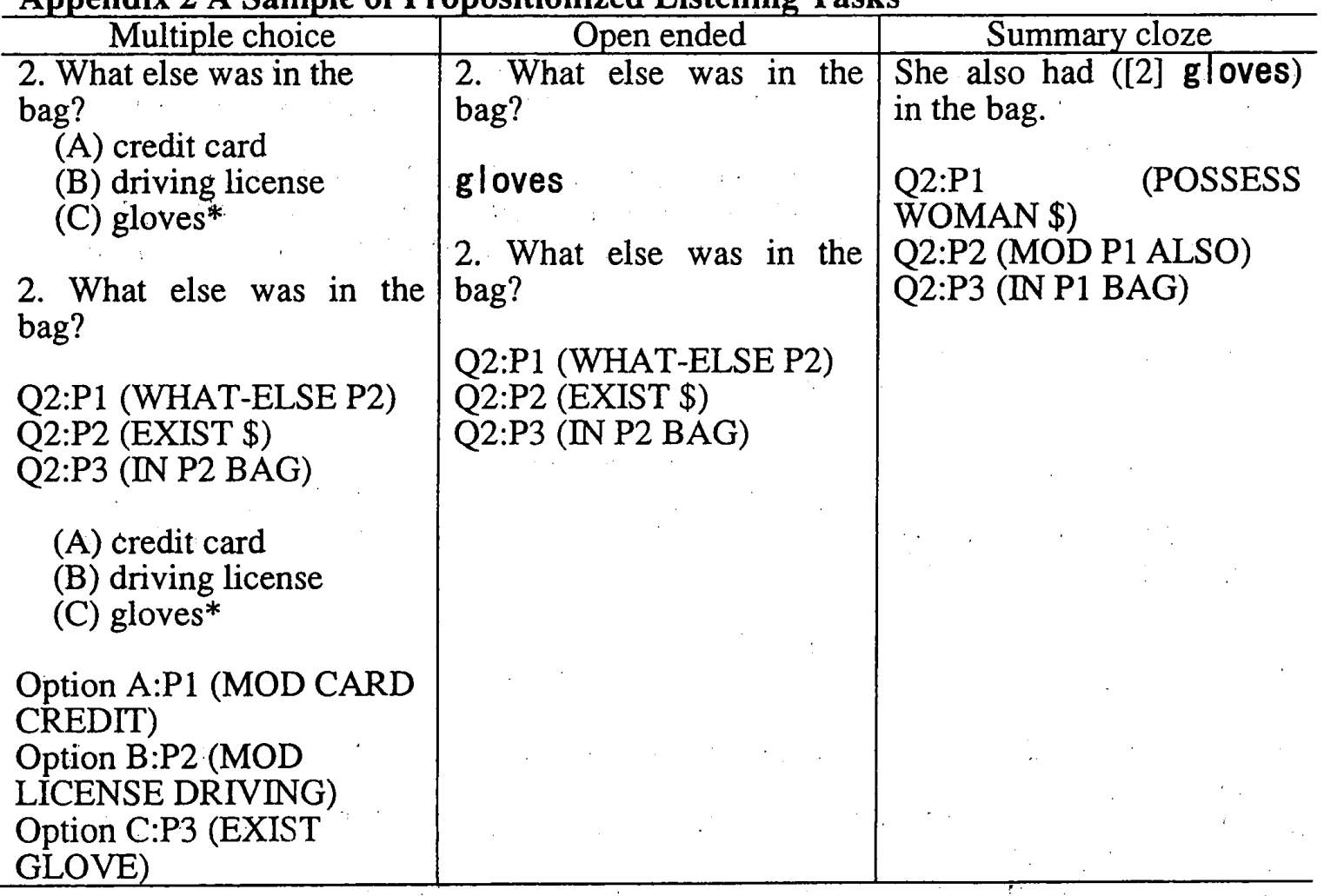

Note. $\mathrm{Q}=$ Question; $\mathrm{S}=$ Sentence; $\mathrm{P}=$ Proposition. 